\title{
Effects of Metformin on the Gut Microbiota in Obesity and Type 2 Diabetes Mellitus
}

This article was published in the following Dove Press journal:

Diabetes, Metabolic Syndrome and Obesity: Targets and Therapy

\author{
Qi Zhang' \\ $\mathrm{Nan} \mathrm{Hu}$ (D) $^{2}$ \\ 'Department of Pharmacy, Changzhou \\ No.7 People's Hospital, Changzhou \\ 213000, People's Republic of China; \\ ${ }^{2}$ Department of Pharmacy, The Third \\ Affiliated Hospital of Soochow University, \\ Changzhou 213000, People's Republic of \\ China
}

\begin{abstract}
Metformin is a first-line treatment for type 2 diabetes mellitus (T2DM); however, its underlying mechanism is not fully understood. Gut microbiota affect the development and progression of T2DM. In recent years, an increasing number of studies has focused on the relationship between metformin and gut microbiota, suggesting that metformin might exert part of its hypoglycemic effect through these microbes. However, most of these results were not consistent due to the complex composition of the microbiota, the differences between species, the large variation between individuals, and the differences in experimental design, bringing great obstacle for our better understanding of the effects of metformin on the gut microbiota. Here, we reviewed the published papers concerning about the impacts of metformin on the gut microbiota of mice, rats, and humans with obesity or T2DM, and summarized the changes of gut microbiota composition caused by metformin and the possible underlying hypoglycemic mechanism which is related to gut microbiota. It was found that the proportions of some microbiota, such as phyla Bacteroidetes and Verrucomicrobia and genera Akkermansia, Bacteroides and Escherichia, were significantly affected by metformin in several studies. Metformin may exert part of hypoglycemic effects by altering the gut microbiota in ways that maintain the integrity of the intestinal barrier, promote the production of short-chain fatty acids (SCFAs), regulate bile acid metabolism, and improve glucose homeostasis.
\end{abstract}

Keywords: metformin, gut microbiota, obesity, type 2 diabetes mellitus

\section{Introduction}

T2DM is a group of clinical syndromes mainly characterized by disorders of glucose metabolism caused by the combined effects of genetic and environmental factors. The significant pathophysiological features of T2DM are the impaired regulatory function of insulin on glucose metabolism (insulin resistance) with reduced (or relatively reduced) insulin secretion caused by pancreatic islet $\beta$-cell dysfunction. ${ }^{1}$ In recent years, with the improvement of people's living standards and dietary changes, the prevalence of obesity and T2DM has increased year by year, and the population with obesity and T2DM has become younger. The latest data from the International Diabetes Federation show that there were approximately 463 million diabetic patients worldwide in 2019. Approximately $87.5 \%$ of the T2DM patients are overweight, according to the 2017 National Diabetes Statistics Report of the CDC. The pathogenesis of T2DM is not fully understood, and it has many similarities with obesity, such as lipid metabolic disorders, stress, and insulin resistance. Obesity, especially the accumulation of abdominal visceral fat, aggravates insulin resistance in patients, which is a major risk factor for the development of T2DM. ${ }^{2,3}$
Department of Pharmacy, The Third

Affiliated Hospital of Soochow University, Changzhou 213000, People's Republic of China

Tel +86-519-68870870

Email hn_324@163.com
Diabetes, Metabolic Syndrome and Obesity: Targets and Therapy 2020:13 5003-5014

5003

DovePress in $\square$ 
Metformin has been used to treat diabetes since the early 1950s and has become a first-line treatment for T2DM. Metformin alone or in combination with other hypoglycemic drugs can effectively control the blood glucose of diabetic patients, especially obese or overweight diabetic patients. The major mechanisms of hypoglycemic action of metformin include inhibiting hepatic gluconeogenesis and reducing hepatic glucose output, increasing glucose uptake and utilization in peripheral tissues (muscle and fat), and improving the energy metabolism in muscle, fat, and liver through the activation of AMP-activated protein kinase. ${ }^{4}$ The oral bioavailability of metformin is approximately $30-60 \%$, the concentration in the intestine is 100-300 times higher than that in the serum, and approximately $50 \%$ of the intake dose is found in the stool. After oral administration, the half-life of metformin is approximately $3-4$ hours, which is inconsistent with the duration of its hypoglycemic effect. ${ }^{5,6}$ In addition, intravenous administration of metformin could not improve blood glucose. These results all suggest that metformin has key effects on the digestive tract.

In recent years, with the development of detection technology, people have realized that the gut microbiota plays an important role in the development and progression of obesity and diabetes. More and more studies focused on relationship between metformin and gut microbiota. However, it is still unclear how metformin affects the gut microbiota as the inconsistent results of the studies. Therefore, we reviewed the literatures concerning the effect of metformin on gut microbiota of mice, rats, and humans with obesity or T2DM, and discussed the hypoglycemic mechanism of metformin which is related to gut microbiota.

\section{T2DM and the Gut Microbiota}

The gut microbiota refers to the microbiota colonizing the surface of the intestinal mucosa. As an environmental factor that is closely associated with the human body, the gut microbiota is involved in human growth and development, physiological processes, and even disease states. There are approximately $10^{14}$ bacteria in the human intestinal tract, which is approximately 10 times the number of human cells throughout the body, and these bacteria carry 100 times the number of genes as the human genome. ${ }^{7}$ These large numbers of bacteria are mainly from nine phyla, Firmicutes, Bacteroidetes, Actinobacteria, Fusobacteria, Proteobacteria, Verrucomicrobia, Cyanobacteria, Spirochaetes, and VadinBE97, among which Firmicutes, Bacteroidetes,
Proteobacteria, and Actinobacteria account for the majority of human gut microbiota. ${ }^{8}$ Intestinal microbes help the body extract nutrients and energy from food. They produce a variety of biologically active compounds, such as vitamins and short-chain fatty acids, and they participate in the regulation of a variety of metabolic processes. ${ }^{9}$

Many studies have analyzed the composition of the gut microbiota of T2DM patients. Larsen et al found differences in the gut microbiota at the phylum level between T2DM patients and normal individuals, eg, the non-T2DM group had a significantly higher proportion of Firmicutes than the T2DM group. ${ }^{10}$ Two metagenome-wide association studies involving relatively large populations were conducted in China and Sweden, confirming the differences in the composition of the gut microbiota between T2DM patients and control groups. ${ }^{11,12}$ Although no consensus had been reached on which bacteria change significantly in T2DM patients, all these studies found a decrease in the number of butyrate-producing bacteria and an increase in conditional pathogens.

It has been found that intestinal microbes participate in the development and progression of T2DM by affecting the integrity of the intestinal barrier, production of SCFAs, and the metabolism of bile acids. Lipopolysaccharide (LPS) is an endotoxin present in the cell wall of gramnegative bacteria that is released after bacterial lysis and can cause a series of nonspecific inflammatory responses. LPS is one of the stressors inducing intestinal mucosal injury and hinders the function of the intestinal barrier. High-fat diet could alter the composition of the gut microbiota in mice and increase the concentration of LPS in mouse plasma, leading to intestinal permeability changes and impaired glucose tolerance. ${ }^{13}$ Changes in the gut microbiota under metabolic disease conditions can downregulate connexins and claudins in intestinal epithelial cells, increase intestinal mucosal permeability, promote LPS release, induce massive production of inflammatory cytokines, and decrease the insulin sensitivity of the body. LPS derived from intestinal microbes is an important factor involved in the development and progression of inflammatory and metabolic diseases. ${ }^{14}$ Gut microbiota can decompose nondigestible food (eg, carbohydrates) into SCFAs. Of these SCFAs, acetic acid, propionic acid, and butyric acid are the most abundant. SCFAs have important metabolic functions and are essential for intestinal health. Butyrate and propionate can activate intestinal gluconeogenesis and have beneficial effects on glucose and energy homeostasis. ${ }^{15}$ SCFAs could also regulate 
glucose metabolism through stimulating the secretion of glucagon-like peptide-1 (GLP-1) and peptide YY in intestinal epithelial L cells. ${ }^{16}$ Yang et al found that the levels of acetic acid, propionic acid, and butyric acid in the stool of diabetic mice were significantly lower than those in the normal and high-fat groups. ${ }^{17}$ Bile acid is the main component of bile, which not only promotes the digestion and absorption of fat but also participates in glycolipid and energy metabolism. Bile acids regulate lipid and glucose metabolism through the activation of the 7-transmembrane bile acid receptor and nuclear farnesoid $\mathrm{X}$ receptor (FXR). ${ }^{18}$ The gut microbiota interacts with bile acids. On the one hand, these bacteria participate in the metabolism of bile acids and transform the primary bile acid of the host by debinding, dehydrogenating, and dehydroxylatingit in the distal small intestine and colon, thereby increasing the diversity of the bile acid pool composition. These modifications can affect the affinity of the bile acids to their receptors. The binding of secondary bile acids to G protein-coupled receptor TGR5 in intestinal epithelial L cells can induce the secretion of GLP-1, regulate the homeostasis of glucose metabolism, and lead to the disorder of glycolipid metabolism. ${ }^{19}$ On the other hand, bile acids also affect the composition of the gut microbiota. ${ }^{20,21}$ For example, secondary bile acids played a role in colonization resistance against Clostridium difficile. ${ }^{22,23}$ The concentrations and compositions of bile acids changed in both T2DM patients and animal models. The postprandial total bile acid (TBA), cholic acid (CA), chenodeoxycholic acid (CDCA), deoxycholic acid (DCA) and ursodeoxycholic acid (UDCA) were higher in T2DM patients than in normal subjects. ${ }^{24}$ The ratio of $12 \alpha-\mathrm{OH}$ to non-12 $\alpha-\mathrm{OH}$ BAs in serum increased in rats with high-fat diet and streptozotocin-induced type 2 diabetes. ${ }^{25}$

\section{Metformin and the Gut Microbiota}

In recent years, with the advancement of gene sequencing technology, an increasing number of studies has focused on the impact of metformin on the gut microbiota. We searched PubMed with the keywords "Metformin AND Gut Microbiota", and pick out all of the studies that clearly described the effect of metformin on composition of gut microbiota in obesity or type 2 diabetes. All these studies were published until 2020. Finally, we found a total of 34 relevant studies and summarized them in Tables 1-3.

A total of fifteen papers were published to investigate the effect of metformin on the gut microbiota in mice with obesity or T2DM (Table 1). C57BL/6 mice were used in most studies, while some studies also studied $\mathrm{db} / \mathrm{db}$ mice, KKAy mice, and ICR mice. In the studies of C57BL/6 mice, the majority of mice were aged 3-8 weeks and were given a high-fat diet (HFD) for different durations (4 days-33 weeks) to induce obesity, followed by metformin treatment $(75-300 \mathrm{mg} / \mathrm{kg} / \mathrm{d}$ ) for 4 days to 14 weeks. $^{26-36}$ Two studies were conducted to study the effect of metformin treatment ( 20 or $113.75 \mathrm{mg} / \mathrm{kg}$ ) for 8 or 11 weeks on diabetic $\mathrm{db} / \mathrm{db}$ mice. ${ }^{37,38}$ Two other studies gave mice a HFD for 3 or 4 weeks to induce obesity in KKAy mice and ICR mice, followed by metformin treatment. ${ }^{39,40}$ Due to the complexity of the gut microbiota and the experimental design of the different studies, such as the different HFD durations and the different dosages and durations of metformin treatment, such studies are bound to have low replicability, but in the published studies, the effects of metformin on certain gut microbiota have been consistent. For example, the proportions of phyla Bacteroidetes ${ }^{27,34,36,39}$ and Verrucomicrobia ${ }^{26,27,29,34,39}$ and genera Akkermansia ${ }^{26,28,29,31,32,34,37-39}$ and Bacteroides $^{29,34,37,39}$ were significantly increased in the metformin treatment group. However, studies in ICR mice showed that the proportion of Bacteroidetes in the gut microbiota of the metformin treatment group was significantly decreased, ${ }^{40}$ suggesting that the effect of metformin on the gut microbiota may be related to the mouse strain.

A total of 9 studies conducted in Wistar rats (five papers), Sprague-Dawley rats (two papers), Otsuka LongEvans Tokushima fatty rats (one paper), and Zucker diabetic fatty rats (one paper) were included (Table 2). Two studies gave the rats HFD for 2 or 10 weeks to induce an obese rat model, ${ }^{41,42}$ five studies treated rats with HFD for 2-12 weeks followed by injection of streptozotocin (STZ) to induce diabetes, ${ }^{43-47}$ and two studies used spontaneously diabetic rat models. ${ }^{48,49}$ The dose of metformin ranged from 30 to $215.12 \mathrm{mg} / \mathrm{kg} / \mathrm{d}$, and the treatment period ranged from 4 weeks to 12 weeks. Similar to the results in mice, Verrucomicrobia was significantly increased in HFD-induced Wistar obese rats after treatment with metformin. ${ }^{41}$ However, in diabetic Wistar rats induced by HFD combined with STZ, metformin treatment showed the opposite effects on Verrucomicrobia. ${ }^{43}$ Like in mice, the increase in genus Akkermansia caused by metformin was also found in rats treated with HFD and diabetic rats. ${ }^{41,45}$

We found ten studies on the effect of metformin on the gut microbiota in obese or T2DM patients, two of which were conducted in obese patients ${ }^{50,51}$ and eight in T2DM patients $^{52-59}$ (Table 3). Among them, four studies 
Table I The Effects of Metformin on Composition of Gut Microbiota in T2DM or Obese Mice

\begin{tabular}{|c|c|c|c|c|c|c|c|}
\hline & $\begin{array}{l}\text { Animal } \\
\text { Model }\end{array}$ & $\begin{array}{l}\text { Control } \\
\text { Group } \\
\text { (Number) }\end{array}$ & $\begin{array}{l}\text { Metformin Treatment } \\
\text { Group (Number) }\end{array}$ & $\begin{array}{l}\text { Effect of Metformin on Gut } \\
\text { Microbiota }\end{array}$ & Country & $\begin{array}{l}\text { Method } \\
\text { for } \\
\text { Detection }\end{array}$ & $\begin{array}{l}\text { Author, } \\
\text { Year and } \\
\text { References }\end{array}$ \\
\hline I & $\begin{array}{l}\text { C57BL/6 } \\
\text { mice }(4 \\
\text { weeks } \\
\text { old) }\end{array}$ & $\begin{array}{l}\text { HFD for } 8 \\
\text { weeks }+ \text { HFD } \\
\text { for } 6 \text { weeks } \\
(n=6)\end{array}$ & $\begin{array}{l}\text { HFD for } 8 \text { weeks }+ \text { HFD } \\
\text { with metformin treatment } \\
(300 \mathrm{mg} / \mathrm{kg} / \mathrm{d}) \text { for } 6 \text { weeks } \\
(\mathrm{n}=6)\end{array}$ & $\begin{array}{l}\text { Phylum: Verrucomicrobia } \uparrow \text { Genus: } \\
\text { Akkermansia } \uparrow\end{array}$ & Korea & I6S rRNA & $\begin{array}{l}\text { Shin et al } \\
2014^{26}\end{array}$ \\
\hline 2 & $\begin{array}{l}\text { C57BL/6 } \\
\text { mice }(6 \\
\text { weeks } \\
\text { old) }\end{array}$ & $\begin{array}{l}\text { HFD for } \\
\text { I8weeks }+ \\
\text { HFD for } \\
\text { IOweeks } \\
(n=N / A)\end{array}$ & $\begin{array}{l}\text { HFD for } 18 \text { weeks }+ \text { HFD } \\
\text { with metformin treatment } \\
(300 \mathrm{mg} / \mathrm{kg} / \mathrm{d}) \text { for } 10 \text { weeks } \\
(\mathrm{n}=\mathrm{N} / \mathrm{A})\end{array}$ & $\begin{array}{l}\text { Phylum: Bacteroidetes } \uparrow, \\
\text { Verrucomicrobia } \uparrow \text { Family: } \\
\text { Bacteroidaceae } \uparrow, \\
\text { Verrucomicrobiaceae } \uparrow, \text { Clostridiales } \\
\text { family XIII } \uparrow \text { Species: Akkermansia } \\
\text { muciniphila } \uparrow, \text { Clostridium cocleatum } \uparrow\end{array}$ & Korea & $\begin{array}{l}\text { I6S rRNA, } \\
\text { qPCR }\end{array}$ & $\begin{array}{l}\text { Lee et al } \\
2014^{27}\end{array}$ \\
\hline 3 & $\begin{array}{l}\text { C57BL/ } \\
6 \mathrm{~J} \text { mice } \\
\text { (3 weeks } \\
\text { old) }\end{array}$ & $\begin{array}{l}\text { HFD for } 12 \\
\text { weeks + HDF } \\
\text { for } 6 \text { weeks } \\
(n=3)\end{array}$ & $\begin{array}{l}\text { HFD for } 12 \text { weeks + HFD } \\
\text { with metformin treatment } \\
(300 \mathrm{mg} / \mathrm{kg} / \mathrm{d}) \text { for } 6 \text { weeks } \\
(\mathrm{n}=3)\end{array}$ & $\begin{array}{l}\text { Genus: Lactococcus, Coprococcus, } \\
\text { Ruminococcus, Staphylococcus, } \\
\text { Akkermansia, Streptococcus, } \\
\text { Oscillospira, Lactobacillus, Allobaculum, } \\
\text { Clostridium, Dorea changed }\end{array}$ & USA & I6S rRNA, & $\begin{array}{l}\text { Bornstein } \\
\text { et al } 2017^{28}\end{array}$ \\
\hline 4 & $\begin{array}{l}\text { Obese } \\
\text { diabetic } \\
\mathrm{db} / \mathrm{db} \\
\text { mice }(8 \\
\text { weeks } \\
\text { old) }\end{array}$ & $\begin{array}{l}\text { Distilled water } \\
\text { for } 8 \text { weeks } \\
(n=10)\end{array}$ & $\begin{array}{l}\text { Metformin treatment } \\
(20 \mathrm{mg} / \mathrm{kg} / \mathrm{d}) \text { for } 8 \text { weeks } \\
(\mathrm{n}=10)\end{array}$ & $\begin{array}{l}\text { Genus: Bacteroidales } \uparrow, \text { Lactobacillus } \uparrow \text {, } \\
\text { Allobaculum } \uparrow, \text { Bacteroides } \uparrow, \\
\text { Akkermansia } \uparrow, \text { Staphylococcus } \downarrow \text {, } \\
\text { Corynebacterium } \downarrow \text {, Jeotgalicoccus } \downarrow \text {, } \\
\text { Aerococcus } \downarrow \text {, Enterococcus } \downarrow \text {, } \\
\text { Facklamia } \downarrow\end{array}$ & China & I6S rRNA & $\begin{array}{l}\text { Chen et al } \\
2018^{37}\end{array}$ \\
\hline 5 & $\begin{array}{l}\text { C57BL/ } \\
6 \mathrm{~N} \text { mice } \\
\text { (6 weeks } \\
\text { old) }\end{array}$ & $\begin{array}{l}\text { HFD for } 23 \\
\text { weeks }+ \text { HFD } \\
\text { for } 16 \text { weeks } \\
(n=6)\end{array}$ & $\begin{array}{l}\text { HFD for } 23 \text { weeks }+ \text { HFD } \\
\text { with metformin treatment } \\
(250 \mathrm{mg} / \mathrm{kg} / \mathrm{d}) \text { for } 16 \\
\text { weeks }(n=6)\end{array}$ & $\begin{array}{l}\text { Phylum: Verrucomicrobia } \uparrow, \\
\text { Firmicutes/Bacteroidetes ratio } \downarrow \\
\text { Genus: Akkermansia } \uparrow, \text { Bacteroides } \uparrow \text {, } \\
\text { Butyricimonas } \uparrow, \text { Parabacteroides } \uparrow\end{array}$ & Korea & $\begin{array}{l}\text { I6S rRNA, } \\
\text { qPCR }\end{array}$ & $\begin{array}{l}\text { Lee et al } \\
2018^{29}\end{array}$ \\
\hline 6 & $\begin{array}{l}\text { KKAy } \\
\text { mice } \\
(8-9 \\
\text { weeks } \\
\text { old) }\end{array}$ & $\begin{array}{l}\text { HFD for } 3 \\
\text { weeks + HFD } \\
\text { with saline } \\
\text { solution for } 8 \\
\text { weeks }(n=6)\end{array}$ & $\begin{array}{l}\text { HFD for } 3 \text { weeks + HFD } \\
\text { with metformin treatment } \\
(314 \mathrm{mg} / \mathrm{kg} / \mathrm{d}) \text { for } 8 \text { weeks } \\
(\mathrm{n}=6)\end{array}$ & $\begin{array}{l}\text { Phylum: Bacteroidetes } \uparrow, \\
\text { Verrucomicrobia } \uparrow, \text { Actinobacteria } \downarrow \text {, } \\
\text { Firmicutes } \downarrow \text { Genus: Bacteroides } \uparrow, \\
\text { Blautia } \uparrow, \text { Escherichia_Shigella } \uparrow, \\
\text { Akkermansia } \uparrow, \text { Aerococcus } \downarrow \text {, } \\
\text { Lactobacillus } \downarrow \text {, Corynebacterium_I }, \\
\text { Staphylococcus } \downarrow\end{array}$ & China & I6S rRNA & $\begin{array}{l}\text { Gao et al } \\
2018^{39}\end{array}$ \\
\hline 7 & $\begin{array}{l}\text { C57BL/ } \\
\text { 6J mice }\end{array}$ & $\begin{array}{l}\text { HFD for } 4 \\
\text { weeks + HFD } \\
\text { for } 10 \text { weeks } \\
(n=8)\end{array}$ & $\begin{array}{l}\text { HFD for } 4 \text { weeks + HFD } \\
\text { with metformin treatment } \\
(100 \mathrm{mg} / \mathrm{kg} / \mathrm{d}) \text { for } 10 \\
\text { weeks }(n=8)\end{array}$ & $\begin{array}{l}\text { Species: Bacteriodetes fragilis } \uparrow \text {, } \\
\text { Escherichia coli } \downarrow\end{array}$ & Korea & $\begin{array}{l}\text { I6S rRNA, } \\
\text { qPCR }\end{array}$ & $\begin{array}{l}\text { Wang et al } \\
2018^{30}\end{array}$ \\
\hline 8 & $\begin{array}{l}\text { C57BL/ } \\
6 \mathrm{~J} \mathrm{mice} \\
\text { ( } 3 \text { weeks } \\
\text { old) }\end{array}$ & $\begin{array}{l}\text { HFD for } 4 \\
\text { weeks }+ \text { STZ+ } \\
\text { HFD with } \\
\text { saline for } 5 \\
\text { weeks }(n=6)\end{array}$ & $\begin{array}{l}\text { HFD for } 4 \text { weeks }+ \text { STZ }+ \\
\text { HFD with metformin } \\
\text { treatment }(200 \mathrm{mg} / \mathrm{kg} / \mathrm{d} \text { or } \\
75 \mathrm{mg} / \mathrm{kg} / \mathrm{d}) \text { for } 5 \text { weeks } \\
(\mathrm{n}=6)\end{array}$ & $\begin{array}{l}\text { Phylum: Firmicutes } \downarrow \text { Genus: } \\
\text { Akkermansia } \uparrow \text { Species: Bacteroides } \\
\text { spp. } \downarrow\end{array}$ & China & I6S rRNA & $\begin{array}{l}\text { Zheng et al } \\
2018^{31}\end{array}$ \\
\hline 9 & $\begin{array}{l}\text { C57BL/ } \\
6 \mathrm{~J} \text { mice } \\
\text { (6 weeks } \\
\text { old) }\end{array}$ & $\begin{array}{l}\text { HFD with } \\
\text { saline for } 3 \\
\text { weeks }(n=5)\end{array}$ & $\begin{array}{l}\text { HFD with metformin } \\
\text { treatment }(300 \mathrm{mg} / \mathrm{kg} / \mathrm{d}) 3 \\
\text { weeks }(\mathrm{n}=5)\end{array}$ & $\begin{array}{l}\text { Genus: Enterococcus, Lactococcus, } \\
\text { Streptococcus, Akkermansia changed }\end{array}$ & China & I6S rRNA & $\begin{array}{l}\text { Ji et al } \\
2019^{32}\end{array}$ \\
\hline
\end{tabular}

(Continued) 
Table I (Continued).

\begin{tabular}{|c|c|c|c|c|c|c|c|}
\hline & $\begin{array}{l}\text { Animal } \\
\text { Model }\end{array}$ & $\begin{array}{l}\text { Control } \\
\text { Group } \\
\text { (Number) }\end{array}$ & $\begin{array}{l}\text { Metformin Treatment } \\
\text { Group (Number) }\end{array}$ & $\begin{array}{l}\text { Effect of Metformin on Gut } \\
\text { Microbiota }\end{array}$ & Country & $\begin{array}{l}\text { Method } \\
\text { for } \\
\text { Detection }\end{array}$ & $\begin{array}{l}\text { Author, } \\
\text { Year and } \\
\text { References }\end{array}$ \\
\hline 10 & $\begin{array}{l}\mathrm{db} / \mathrm{db} \\
\text { mice }(8 \\
\text { weeks } \\
\text { old) }\end{array}$ & $\begin{array}{l}\text { Saline solution } \\
\text { for II weeks } \\
(n=5)\end{array}$ & $\begin{array}{l}\text { Metformin treatment } \\
(\text { I } 13.75 \mathrm{mg} / \mathrm{kg} / \mathrm{d}) \text { for II } \\
\text { weeks }(n=5)\end{array}$ & $\begin{array}{l}\text { Genus: Butyricimonas } \uparrow, \text { Lactobacillus } \uparrow \\
\text { Coprococcus } \uparrow, \text { Ruminococcus } \uparrow \\
\text { Akkermansia } \uparrow \text { Genus: Prevotella } \downarrow \text {, } \\
\text { Proteus } \downarrow\end{array}$ & China & $\begin{array}{l}\text { I6S rRNA, } \\
\text { qPCR }\end{array}$ & $\begin{array}{l}\text { Zhang et al } \\
2019^{38}\end{array}$ \\
\hline 11 & $\begin{array}{l}\text { ICR mice } \\
\text { (8 weeks } \\
\text { old) }\end{array}$ & $\begin{array}{l}\text { HFD+STZ } \\
+ \text { HFD for } 4 \\
\text { weeks }(n=10)\end{array}$ & $\begin{array}{l}\text { HFD+STZ+HFD with } \\
\text { metformin treatment } \\
(200 \mathrm{mg} / \mathrm{kg} / \mathrm{d}) \text { for } 4 \text { weeks } \\
(\mathrm{n}=10)\end{array}$ & $\begin{array}{l}\text { Phylum: Firmicutes } \uparrow, \text { Bacteroidetes } \downarrow \text {, } \\
\text { Proteobacteria } \downarrow \text { Species: Lactobacillus } \\
\text { spp. } \uparrow, \text { Lactobacillales } \uparrow\end{array}$ & China & I6S rRNA & $\begin{array}{l}\text { Chen et al } \\
2019^{40}\end{array}$ \\
\hline 12 & $\begin{array}{l}\text { C57BL/ } \\
6 \mathrm{~J} \mathrm{mice} \\
(6-8 \\
\text { weeks } \\
\text { old) }\end{array}$ & $\begin{array}{l}\text { FFC for } 4 \text { days } \\
(n=6)\end{array}$ & $\begin{array}{l}\text { FFC with metformin } \\
\text { treatment }(300 \mathrm{mg} / \mathrm{kg} / \mathrm{d}) \\
\text { for } 4 \text { days }(\mathrm{n}=6)\end{array}$ & $\begin{array}{l}\text { Species: Lactobacillus animalis } \downarrow \text {, } \\
\text { Unclassifed members of Burkholderia } \\
\text { and Romboutsia genera } \downarrow \text {, An } \\
\text { unclassified member of Alloprevotella } \\
\text { family } \downarrow\end{array}$ & Austria & I6S rRNA & $\begin{array}{l}\text { Brandt et al } \\
2019^{33}\end{array}$ \\
\hline 13 & $\begin{array}{l}\text { C57BL/6 } \\
\text { mice }(3 \\
\text { weeks } \\
\text { old) }\end{array}$ & $\begin{array}{l}\text { HFD for } 12 \\
\text { weeks }+ \text { HFD } \\
\text { for } 12 \text { weeks } \\
(n=14)\end{array}$ & $\begin{array}{l}\text { HFD for } 12 \text { weeks + HFD } \\
\text { with metformin treatment } \\
(300 \mathrm{mg} / \mathrm{kg} / \mathrm{d}) \text { for } 12 \\
\text { weeks }(n=14)\end{array}$ & $\begin{array}{l}\text { Phylum: Bacteroidetes } \uparrow, \\
\text { Verrucomicrobia } \uparrow \text {, Firmicutes/ } \\
\text { Bacteroidetes ratio } \downarrow \text { Genus: } \\
\text { Bacteroides } \uparrow, \text { Akkermansia } \uparrow, \\
\text { Parabacteroides } \uparrow, \text { Christensenella } \uparrow, \\
\text { Clostridiales other } \uparrow, \text { AF-I } 2 \downarrow \text {, } \\
\text { Muribaculum } \downarrow \text {, Clostridiaceae other } \downarrow \text {, } \\
\text { Lachnoclostridium } \downarrow \text {, Coprococcus } \downarrow \text {, } \\
\text { Dorea } \downarrow \text {, Papillibacter } \downarrow \text {, Oscillospira } \downarrow \text {, } \\
\text { Ruminococcus } \downarrow \text {, Desulfovibrio } \downarrow \text {, } \\
\text { Desulfovibrionaceae } \downarrow\end{array}$ & Ireland & I6S rRNA & $\begin{array}{l}\text { Ryan et al } \\
2020^{34}\end{array}$ \\
\hline 14 & $\begin{array}{l}\text { C57BL/ } \\
\text { 6J mice } \\
\text { (5 weeks } \\
\text { old) }\end{array}$ & $\begin{array}{l}\text { HFD for } 14 \\
\text { weeks }(n=12)\end{array}$ & $\begin{array}{l}\text { HFD with metformin } \\
\text { treatment }(200 \mathrm{mg} / \mathrm{kg} / \mathrm{d}) \\
\text { for } 14 \text { weeks }(n=12)\end{array}$ & Family: Lachnospiraceae $\downarrow$ & USA & I6S rRNA & $\begin{array}{l}\text { Chung et al } \\
2020^{35}\end{array}$ \\
\hline 15 & $\begin{array}{l}\text { C57BL/ } \\
6 \mathrm{~J} \text { mice } \\
(78 \\
\text { weeks } \\
\text { old) }\end{array}$ & $\begin{array}{l}\text { HFD for II } \\
\text { weeks }(n=5-7)\end{array}$ & $\begin{array}{l}\text { HFD with metformin } \\
\text { treatment }(100 \mathrm{mg} / \mathrm{kg} / \mathrm{d}) \\
\text { for II weeks }(\mathrm{n}=5-7)\end{array}$ & $\begin{array}{l}\text { Phylum: Bacteroidetes } \uparrow \text { Genus: } \\
\text { Genera belonging to S24_7, } \\
\text { Ruminococcaceae, and Lactococcus } \uparrow \text {, } \\
\text { Genera belonging to Veilonellaceae, } \\
\text { Coriobacteriaceae, Lactobacillus, Dorea, } \\
\text { SMB53, Roseburia, and } \\
\text { Dehalobacterium } \downarrow\end{array}$ & USA & I6S rRNA & $\begin{array}{l}\text { Ahmadi et al } \\
2020^{36}\end{array}$ \\
\hline
\end{tabular}

Notes: $\uparrow$, Increase; $\downarrow$, decrease.

Abbreviations: N/A, not available; STZ, streptozotozin; HFD, high-fat diet; FFC, fat-fructose- and cholesterol-rich diet.

compared the changes in the gut microbiota of newly diagnosed T2DM patients (without drug treatment before the visit) who received metformin for different follow-up times (from 3 days to 2 years). Four studies were conducted in T2DM patients who had received drug treatment and compared the gut microbiota of patients with vs without metformin treatment. The results in humans are quite different from the results of animal studies. Clinical trials on diabetic patients are affected by many complex factors, such as experimental design, diet, ethnic origins, course of disease, comorbidities, and combined medications. In addition, there were significant differences in intestinal microbial diversity between humans and animals. ${ }^{60}$ Results of some bacteria were consistent in humans and 
Table 2 The Effects of Metformin on Composition of Gut Microbiota in T2DM or Obese Rats

\begin{tabular}{|c|c|c|c|c|c|c|c|}
\hline & $\begin{array}{l}\text { Animal } \\
\text { Model }\end{array}$ & $\begin{array}{l}\text { Control Group } \\
\text { (Number) }\end{array}$ & $\begin{array}{l}\text { Metformin Treatment } \\
\text { Group (Number) }\end{array}$ & $\begin{array}{l}\text { Effect of Metformin on Gut } \\
\text { Microbiota }\end{array}$ & Country & $\begin{array}{l}\text { Method } \\
\text { for } \\
\text { Detection }\end{array}$ & $\begin{array}{l}\text { Author, } \\
\text { Year and } \\
\text { References }\end{array}$ \\
\hline I & $\begin{array}{l}\text { Wistar } \\
\text { rats (8-10 } \\
\text { weeks } \\
\text { old) }\end{array}$ & $\begin{array}{l}\text { HFD for } 10 \text { weeks } \\
+ \text { HFD for } 8 \text { weeks } \\
(n=10)\end{array}$ & $\begin{array}{l}\text { HFD for } 10 \text { weeks }+ \\
\text { HFD with metformin } \\
\text { treatment }(200 \mathrm{mg} / \mathrm{kg} / \mathrm{d}) \\
\text { for } 8 \text { weeks }(n=10)\end{array}$ & 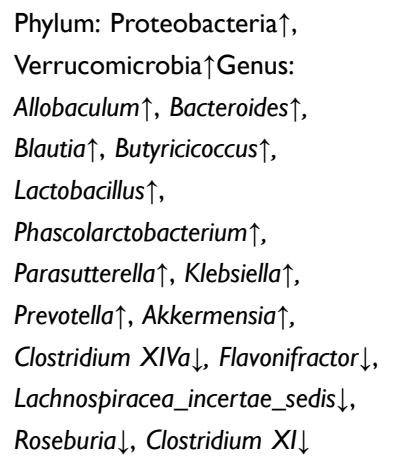 & China & I6S rRNA & $\begin{array}{l}\text { Zhang et al } \\
2015^{41}\end{array}$ \\
\hline 2 & $\begin{array}{l}\text { OLETF } \\
\text { rats (4 } \\
\text { weeks } \\
\text { old) }\end{array}$ & $\begin{array}{l}\text { Distilled water for } \\
12 \text { weeks }(n=7)\end{array}$ & $\begin{array}{l}\text { Metformin treatment } \\
(100 \mathrm{mg} / \mathrm{kg} / \mathrm{d}) \text { for } 12 \\
\text { weeks }(\mathrm{n}=7)\end{array}$ & $\begin{array}{l}\text { Species: Akkermansia spp. } \uparrow, \\
\text { Prevotella spp. } \downarrow\end{array}$ & Korea & $\begin{array}{l}\text { DGGE, } \\
\text { qPCR }\end{array}$ & $\begin{array}{l}\text { Wang et al } \\
2017^{48}\end{array}$ \\
\hline 3 & $\begin{array}{l}\text { Wistar } \\
\text { rats } \\
(200 \mathrm{~g})\end{array}$ & $\begin{array}{l}\text { HFD for } 4 \text { weeks }+ \\
\text { STZ + HFD for } 2 \\
\text { weeks +HFD for } 4 \\
\text { weeks }(n=8)\end{array}$ & $\begin{array}{l}\text { HFD for } 4 \text { weeks }+ \text { STZ } \\
+ \text { HFD for } 2 \text { weeks + } \\
\text { HFD with metformin } \\
\text { treatment }(200 \mathrm{mg} / \mathrm{kg} / \mathrm{d}) \\
\text { for } 4 \text { weeks }(n=9)\end{array}$ & 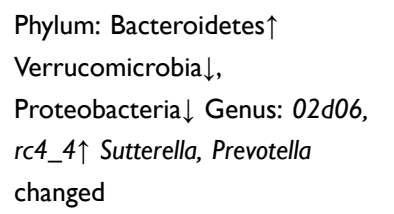 & China & $\begin{array}{l}\text { I6S rRNA, } \\
\text { qPCR }\end{array}$ & $\begin{array}{l}\text { Liu et al } \\
2018^{43}\end{array}$ \\
\hline 4 & $\begin{array}{l}\text { Wistar } \\
\text { rats } \\
(200 \mathrm{~g})\end{array}$ & $\begin{array}{l}\text { HFD for } 4 \text { weeks }+ \\
\text { STZ + HFD for } 2 \\
\text { weeks +HDF for } 4 \\
\text { weeks }(n=6)\end{array}$ & $\begin{array}{l}\text { HFD for } 4 \text { weeks }+ \text { STZ } \\
+ \text { HFD for } 2 \text { weeks } \\
+ \text { HFD wit metformin } \\
\text { treatment }(200 \mathrm{mg} / \mathrm{kg} / \mathrm{d}) \\
\text { for } 4 \text { weeks }(n=7)\end{array}$ & Phylum: Bacteroidetes changed & China & I6S rRNA & $\begin{array}{l}\text { Liu et al } \\
2018^{44}\end{array}$ \\
\hline 5 & $\begin{array}{l}\text { Zucker } \\
\text { diabetic } \\
\text { fatty fa/fa } \\
\text { (ZDF) } \\
\text { rats (7 } \\
\text { weeks } \\
\text { old) }\end{array}$ & $\begin{array}{l}\text { HFD for } 4 \text { weeks }+ \\
\text { HFD with saline for } \\
4 \text { weeks }(n=8)\end{array}$ & $\begin{array}{l}\text { HFD for } 4 \text { weeks + HFD } \\
\text { with metformin } \\
\text { treatment }(215.15 \mathrm{mg} / \mathrm{kg} / \\
\text { d) for } 4 \text { weeks }(n=8)\end{array}$ & $\begin{array}{l}\text { Phylum: Firmicutes } \uparrow \text { Genus: } \\
\text { Lactobacillus } \uparrow \text { Species: } \\
\text { Lactobacillus intestinalis } \uparrow \text {, } \\
\text { Lactobacillus johnsonii } \uparrow\end{array}$ & China & $16 \mathrm{~S}$ rRNA & $\begin{array}{l}\text { Zhang et al } \\
2019^{49}\end{array}$ \\
\hline 6 & $\begin{array}{l}\text { Wistar } \\
\text { rats }\end{array}$ & $\begin{array}{l}\text { HFD for } 4 \text { months } \\
+ \text { HFD with saline } \\
\text { for } 6 \text { weeks }(n=8)\end{array}$ & $\begin{array}{l}\text { HFD for } 4 \text { months +HFD } \\
\text { with metformin } \\
\text { treatment }(150 \mathrm{mg} / \mathrm{kg} / \mathrm{d}) \\
\text { for } 6 \text { weeks }(\mathrm{n}=8)\end{array}$ & $\begin{array}{l}\text { Species: Bifidobacteria } \uparrow, \\
\text { Akkermansia } \uparrow, \text { Shewanella } \uparrow, \\
\text { Allobaculum } \uparrow, \\
\text { Peptostreptococcaceae } \uparrow, \\
\text { Intestinibacter } \uparrow, \text { Prevotella } \downarrow \text {, } \\
\text { Deferribacteres } \downarrow\end{array}$ & China & $16 \mathrm{~S}$ rRNA & $\begin{array}{l}\text { Li et al } \\
2019^{42}\end{array}$ \\
\hline 7 & $\begin{array}{l}\text { Sprague- } \\
\text { Dawley } \\
\text { rats (100- } \\
120 \mathrm{~g})\end{array}$ & $\begin{array}{l}\text { HFD for } 12 \text { weeks } \\
+ \text { STZ + HFD for } 2 \\
\text { days + HFD with } \\
\text { distilled water for } \\
\text { 6weeks }(n=6)\end{array}$ & $\begin{array}{l}\text { HFD for } 12 \text { weeks + STZ } \\
+ \text { HFD for } 2 \text { days + HFD } \\
\text { with metformin } \\
\text { treatment }(100 \mathrm{mg} / \mathrm{kg} / \mathrm{d}) \\
\text { for } 6 \text { weeks }(\mathrm{n}=6)\end{array}$ & 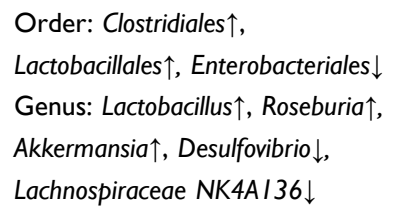 & China & $16 \mathrm{~S}$ rRNA & $\begin{array}{l}\text { Cui et al } \\
2019^{45}\end{array}$ \\
\hline
\end{tabular}

(Continued) 
Table 2 (Continued).

\begin{tabular}{|c|c|c|c|c|c|c|c|}
\hline & $\begin{array}{l}\text { Animal } \\
\text { Model }\end{array}$ & $\begin{array}{l}\text { Control Group } \\
\text { (Number) }\end{array}$ & $\begin{array}{l}\text { Metformin Treatment } \\
\text { Group (Number) }\end{array}$ & $\begin{array}{l}\text { Effect of Metformin on Gut } \\
\text { Microbiota }\end{array}$ & Country & $\begin{array}{l}\text { Method } \\
\text { for } \\
\text { Detection }\end{array}$ & $\begin{array}{l}\text { Author, } \\
\text { Year and } \\
\text { References }\end{array}$ \\
\hline 8 & $\begin{array}{l}\text { Sprague- } \\
\text { Dawley } \\
\text { rats (I70- } \\
\text { 190g) }\end{array}$ & $\begin{array}{l}\text { HFD for } 6 \text { weeks }+ \\
\text { STZ + HFD for I } \\
\text { week + HFD with } \\
\text { saline for 8weeks } \\
(n=6)\end{array}$ & $\begin{array}{l}\text { HFD for } 6 \text { weeks }+ \text { STZ } \\
+ \text { HFD for I week + } \\
\text { HFD with metformin } \\
\text { treatment }(120 \mathrm{mg} / \mathrm{kg} / \mathrm{d}) \\
\text { for } 8 \text { weeks }(\mathrm{n}=6)\end{array}$ & $\begin{array}{l}\text { Genus: } \\
\text { Eubacterium_xylanophilum } \uparrow, \\
\text { Oscillibacter } \uparrow, \text { Anaerotruncus } \uparrow, \\
\text { Bacteroidales_S24-7_group } \downarrow\end{array}$ & China & I6s rRNA & $\begin{array}{l}\text { Liu et al } \\
2020^{46}\end{array}$ \\
\hline 9 & $\begin{array}{l}\text { Wistar } \\
\text { rats (180- } \\
200 \mathrm{~g})\end{array}$ & $\begin{array}{l}\text { HFD for } 2 \text { weeks }+ \\
\text { STZ + HFD for } 2 \\
\text { weeks }+ \text { HFD for } \\
\text { I2weeks }(n=5)\end{array}$ & $\begin{array}{l}\text { HFD for } 2 \text { weeks }+ \text { STZ } \\
+ \text { HFD for } 2 \text { weeks }+ \\
\text { HFD with metformin } \\
\text { treatment }(30 \mathrm{mg} / \mathrm{kg} / \mathrm{d}) \\
\text { for } 12 \text { weeks }(n=5)\end{array}$ & $\begin{array}{l}\text { Species: Lactobacillus spp. } \uparrow \text {, } \\
\text { Bifidobacterium spp. } \uparrow, \text { Escherichia } \\
\text { spp. } \downarrow, \text { Clostridium perfringens } \downarrow\end{array}$ & Thailand & qPCR & $\begin{array}{l}\text { Khat- } \\
\text { udomkii } \\
\text { et al } 2020^{47}\end{array}$ \\
\hline
\end{tabular}

Notes: $\uparrow$, Increase; $\downarrow$, decrease.

Abbreviations: N/A, not available; STZ, streptozotozin; DGGE, denaturing gradient gel electrophoresis.

rodents. Hiel et al reported that the proportion of Akkermansia significantly increased in obese patients on metformin, which was consistent with the results in animal studies. ${ }^{50}$ The proportion of Akkermansia muciniphila was also increased in metformin-treated T2DM patients. ${ }^{54}$ Forslund et al found that Escherichia spp. significantly increased in T2DM patients treated with metformin over those not treated with metformin. ${ }^{52}$ Similarly, Wu et al showed that Escherichia significantly increased in newly diagnosed T2DM patients after treatment with metformin. ${ }^{53}$ Two studies on obese patients also found that metformin significantly increased the proportion of the Escherichia/Shigella group. ${ }^{50,51}$

Dysbiosis in gut microbiota is strongly associated with the pathogenesis of obesity and T2DM. The composition of gut microbiota exhibited significant differences among obese, T2DM and healthy individuals. ${ }^{61,62}$ High fat diet and STZ induction change the gut microbiota of rats or mice. ${ }^{63,64}$ Besides, metformin also had different effects on the gut microbiota of obese/diabetic and healthy humans/ animals. Metformin decreased the bacterial diversity in mice fed a high-fat diet, but had no effect on bacterial diversity in mice fed a normal diet. ${ }^{27}$ The various influencing factors make the change of gut microbiota more complex. In order to better understand the gut microbiota in the clinical use of metformin, we just reviewed the literatures concerning the impacts of metformin on composition of gut microbiota in T2DM or obese rodents or patients.

There were also some studies which investigate the effect of metformin on gut microbiota in healthy subjects or normal rats/mice. The short-term effect of metformin on composition of gut microbiota has been evaluated in healthy Caucasian volunteers, and seven days of treatment with metformin was associated with a significant decrease in the families Peptostreptococcaceae and Clostridiaceae_1 and four genera within these families. ${ }^{65}$ Another clinical trial conducted in 27 healthy young Danish men showed that metformin reduced the abundance of Intestinibacter spp. and Clostridium spp., while increased the abundance of Escherichia/Shigella spp. and Bilophila wadsworthia. ${ }^{66}$ Metformin also altered the gut microbiota of healthy mice, and it was found that microbes from the Verrucomicrobiaceae and Prevotellaceae classes were enriched, while those from Lachnospiraceae and Rhodobacteraceae were depleted. ${ }^{67}$

\section{The Mechanisms by Which Metformin Exerts Hypoglycemic Effects Through the Gut Microbiota}

\section{Maintaining Intestinal Barrier Integrity}

The interaction between the intestinal barrier integrity and the gut microbiota is very important in the development and progression of metabolic diseases. Shin et al found that metformin inhibited the increase in serum LPS induced by HFD and believed that it was due to the change in the gut microbiota composition. ${ }^{26}$ Similarly, metformin reduced the blood LPS level and protected intestinal barrier function in a HFD-induced mouse model of obesity and insulin resistance. ${ }^{68}$ In addition, exogenous administration of LPS can block the enhancement of blood 
Table 3 The Effects of Metformin on Composition of Gut Microbiota in T2DM or Obese Patients

\begin{tabular}{|c|c|c|c|c|c|c|}
\hline & $\begin{array}{l}\text { Control Group } \\
\text { (Number) }\end{array}$ & $\begin{array}{l}\text { Metformin Treatment } \\
\text { Group (Number) }\end{array}$ & Effect of Metformin on Gut Microbiota & Country & $\begin{array}{l}\text { Method for } \\
\text { Detection }\end{array}$ & $\begin{array}{l}\text { Author, } \\
\text { Year and } \\
\text { References }\end{array}$ \\
\hline I & $\begin{array}{l}\text { Metformin- } \\
\text { untreated T2DM } \\
\text { patients }(n=106)\end{array}$ & $\begin{array}{l}\text { Metformin-treated T2DM } \\
\text { patients }(n=93)\end{array}$ & Species: Escherichia spp. $\uparrow$, Intestinibacter spp. $\downarrow$ & Germany & Metagenomic & $\begin{array}{l}\text { Forslund } \\
\text { et al } 2015^{52}\end{array}$ \\
\hline 2 & $\begin{array}{l}\text { Metformin- } \\
\text { untreated T2DM } \\
\text { patients }(n=14)\end{array}$ & $\begin{array}{l}\text { Metformin-treated T2DM } \\
\text { patients }(n=14)\end{array}$ & 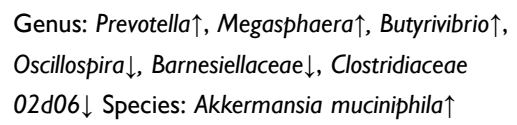 & Colombia & I6S rRNA & $\begin{array}{l}\text { Cuesta- } \\
\text { Zuluaga et al } \\
2017^{54}\end{array}$ \\
\hline 3 & $\begin{array}{l}\text { Treatment-naive } \\
\text { newly diagnosed } \\
\text { T2DM }(n=22)\end{array}$ & $\begin{array}{l}\text { Treatment-naive newly } \\
\text { diagnosed T2DM }+4 \text { months } \\
\text { of metformin treatment } \\
(1700 \mathrm{mg} / \mathrm{d})(\mathrm{n}=22)\end{array}$ & 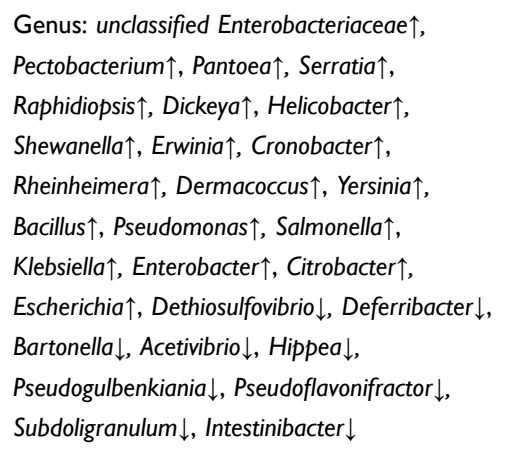 & Sweden & Metagenomic & $\begin{array}{l}\text { Wu et al } \\
2017^{53}\end{array}$ \\
\hline 4 & $\begin{array}{l}\text { Metformin- } \\
\text { untreated T2DM } \\
\text { patients }(n=23)\end{array}$ & $\begin{array}{l}\text { Metformin-treated T2DM } \\
\text { patients }(n=23)\end{array}$ & Family: Enterobacteriaceae $\uparrow$ & Sweden & $\begin{array}{l}\text { qPCR, } \\
\text { T-RFLP }\end{array}$ & $\begin{array}{l}\text { Hung et al } \\
2018^{56}\end{array}$ \\
\hline 5 & $\begin{array}{l}\text { Treatment-naive } \\
\text { newly diagnosed } \\
\text { T2DM }(n=22)\end{array}$ & $\begin{array}{l}\text { Treatment-naive newly } \\
\text { diagnosed T2DM }+3 \text { days of } \\
\text { metformin treatment } \\
(1000 \mathrm{mg} \text { b.i.d })(n=22)\end{array}$ & $\begin{array}{l}\text { Genus: Bacteroides } \downarrow \text { Species: Bacteroides } \\
\text { fragilis } \downarrow\end{array}$ & China & Metagenomic & $\begin{array}{l}\text { Sun et al } \\
2018^{55}\end{array}$ \\
\hline 6 & $\begin{array}{l}\text { African American } \\
\text { men with T2DM } \\
\text { who do not take } \\
\text { metformin }(n=11)\end{array}$ & $\begin{array}{l}\text { African American men with } \\
\text { T2DM who take metformin } \\
(n=21)\end{array}$ & $\begin{array}{l}\text { Genus: Bifidobacterium } \uparrow, \text { Catenibacterium } \uparrow, \\
\text { Parabacteroides } \uparrow\end{array}$ & USA & $16 \mathrm{~S}$ rRNA & $\begin{array}{l}\text { Barengolts } \\
\text { et al } 2018^{57}\end{array}$ \\
\hline 7 & $\begin{array}{l}\text { Obese women } \\
\text { before metformin } \\
\text { treatment }(n=20)\end{array}$ & $\begin{array}{l}\text { Obese women after } 2 \\
\text { months of low-calorie diet } \\
\text { and metformin treatment } \\
(1000 \mathrm{mg} / \mathrm{d})(\mathrm{n}=20)\end{array}$ & Genus: Escherichia/Shigella $\uparrow$ & Iran & I6S rRNA & $\begin{array}{l}\text { Ejtahed et al } \\
2019^{51}\end{array}$ \\
\hline 8 & $\begin{array}{l}\text { T2DM patients } \\
\text { before metformin } \\
\text { treatment }(n=26)\end{array}$ & $\begin{array}{l}\text { T2DM patients after more } \\
\text { than } 3 \text { months of metformin } \\
\text { treatment }(n=5 I)\end{array}$ & $\begin{array}{l}\text { Phylum: Actinobacteria } \uparrow, \text { Fusobacteria } \uparrow \text { Class: } \\
\text { Betaproteobacteria } \downarrow \text {, } \\
\text { Gammaproteobacteria } \downarrow \text { Erysipelotrichi } \downarrow \text { Genus: } \\
\text { Turicibacter } \uparrow, \text { Fusobacterium } \uparrow\end{array}$ & China & 16S rRNA & $\begin{array}{l}\text { Zhang et al } \\
2019^{58}\end{array}$ \\
\hline 9 & $\begin{array}{l}\text { Metformin-naive } \\
\text { obese patients } \\
(n=53)\end{array}$ & $\begin{array}{l}\text { Metformin-treated obese } \\
\text { patients }(n=42)\end{array}$ & 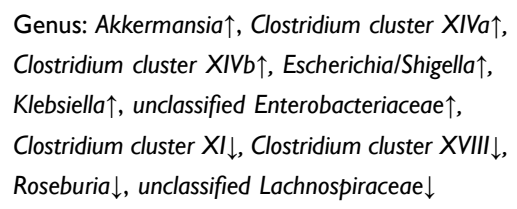 & Belgium & I6S rRNA & $\begin{array}{l}\text { Hiel et al } \\
2020^{50}\end{array}$ \\
\hline 10 & $\begin{array}{l}\text { Treatment-naive } \\
\text { T2DM patients } \\
(n=14)\end{array}$ & $\begin{array}{l}\text { T2DM patients after } I-2 \\
\text { years of metformin } \\
\text { treatment }(n=14)\end{array}$ & $\begin{array}{l}\text { Order: Bacteroidales } \uparrow, \text { Acidobacteriales } \uparrow \text { Family: } \\
\text { Koribacteraceae } \uparrow \text { Genus: Pelomonas spp. } \uparrow\end{array}$ & Mexico & I6S rRNA & $\begin{array}{l}\text { Chávez- } \\
\text { Carbajal } \\
\text { et al } 2020^{59}\end{array}$ \\
\hline
\end{tabular}

Notes: $\uparrow$, Increase; $\downarrow$, decrease.

Abbreviations: N/A, not available; T-RFLP, terminal restriction fragment length polymorphism. 
glucose control and insulin signaling caused by metformin. These results indicate that metformin might improve glucose metabolism by maintaining intestinal barrier function.

\section{Promotion of SCFA Production}

SCFAs produced by the gut microbiota can exert beneficial effects on peripheral tissues, such as adipose tissue, skeletal muscle, and liver, by controlling substrate metabolism and function, thereby improving insulin sensitivity. The ability of the gut microbiota to produce butyrate and propionate is enhanced in patients treated with metformin. Metformin treatment can increase the abundance of SCFAproducing bacteria in mice and rats, such as Allobaculum, Bacteroides, Blautia, Butyricicoccus, Lactobacillus, Akkermansia, Phascolarctobacterium, Butyricimonas, Coprococcus, and Ruminococcus. ${ }^{26,27,29,38,41}$ A similar finding was observed in T2DM patients: after taking metformin, the relative abundance of SCFA-producing bacteria increased (eg, Akkermansia, Lactobacillus, Bifidobacterium, Prevotella, Megasphaera, Shewanella, Blautia, and Butyrivibrio). ${ }^{52-54}$

\section{Regulation of Bile Acid Metabolism}

It was found that metformin can improve glucose metabolism by regulating the total bile acid level in the serum of diabetic rats. $\mathrm{Wu}$ et al showed that metformin treatment for 4 months significantly increased plasma bile acid, while bile acid in stool samples remained unchanged. In the second month of metformin treatment, the level of bile salt hydrolase (bsh) produced by the gut microbiota increased. ${ }^{53}$ Sun et al analyzed the gut microbiota in patients who were newly diagnosed with T2DM and were treated for the first time with metformin for 3 days. They found that metformin increased the level of glycoursodeoxycholic acid (GUDCA) by regulating the gut microbiota (such as inhibition of Bacteroides fragilis growth), thereby inhibiting the FXR signaling pathway to reduce blood glucose and maintain blood glucose homeostasis. ${ }^{55}$ It was found that metformin reduced the reabsorption of bile acid in the distal ileum, leading to the increasing bile salt concentration within the colon, which might account for the effects of metformin on the colonic microbiota. ${ }^{69,70}$

\section{Regulating Specific Bacteria to Maintain Glucose Homeostasis}

Through summarizing and comparing the results in patients and rodent models, we found that metformin significantly increases the abundance of the phylum Verrucomicrobia, genus Akkermansia, and species Akkermansia muciniphila. ${ }^{26,27,31,41,42,45,48,53,54}$ Zhang et al found that Akkermansia muciniphila was reduced in patients with prediabetes (impaired glucose tolerance and/ or impaired fasting glucose) and newly diagnosed T2DM patients, suggesting that a low abundance of this bacterium might be a biomarker of glucose intolerance. ${ }^{71}$ Lee et al found that Akkermansia muciniphila was negatively correlated with serum glucose level in HFD-fed mice treated with metformin. ${ }^{27}$ Akkermansia muciniphila is one of the most abundant single species in the human gut microbiota, a well-known utilizer of mucin, and has been negatively correlated with obesity, diabetes, and cardiovascular diseases. ${ }^{72}$ It plays an important role in maintaining the integrity of the mucosa and could reduce the migration of the pro-inflammatory LPS and improve blood glucose. ${ }^{73}$

\section{Other Possible Mechanisms}

It was reported by Horakova et al that metformin decreased glucose transport from the intestinal lumen into the blood. ${ }^{74}$ Morita et al evaluated the accumulation of $\left[{ }^{18} \mathrm{~F}\right]$ fluorodeoxyglucose in different portions of the intestine from 24 pairs of patients with T2DM who were receiving treatment with metformin or were not. ${ }^{75}$ The results suggested that metformin could promote the transport of glucose from the circulation into stool. Bauer et al proposed a new mechanism of the hypoglycemic effect of metformin associated with the gut microbiota in rodents. ${ }^{76}$ It was showed that high-fat diet changed the upper small intestinal microbiota and compromised glucose sensing in rats. Metformin treatment in the upper small intestine restored sodium-glucose cotransporter 1 (SGLT1) expression and glucose sensing while shifting the upper small intestinal microbiota partly by increasing the abundance of Lactobacillus. In addition, metformin-treated microbiota transplants restored glucose SGLT1 sensing.

\section{Conclusions}

Metformin has been shown to alter the composition of the gut microbiota in mouse and rat models induced by HFD and in T2DM patients, manifested as changes in the relative proportions of certain bacteria of different taxonomic levels. Metformin can improve glucose homeostasis and exert hypoglycemic effects by affecting the gut microbiota, through which it maintains intestinal barrier function, increases the production of SCFAs, regulates bile acid metabolism, and affects glucose homeostasis. The relationship among T2DM, 
metformin, and the gut microbiota is gradually being understood, but the results of different studies are slightly different. It is still unclear how much of the hypoglycemic effect of metformin comes from its alteration of the gut microbiota and what role the microbiota plays in the development and progression of T2DM. Therefore, we expect future in-depth studies to take on the gut microbiota as a new hypoglycemic target.

\section{Funding}

This study was funded by the National Science Foundation of China (No: 81503136) and the Changzhou High-Level Medical Talents Training Project (No: 2016CZBJ010).

\section{Disclosure}

The authors declare no conflict of interest.

\section{References}

1. Pearson ER. Type 2 diabetes: a multifaceted disease. Diabetologia. 2019;62(7):1107-1112. doi:10.1007/s00125-019-4909-y

2. Caspard H, Jabbour S, Hammar N, Fenici P, Sheehan JJ, Kosiborod M. Recent trends in the prevalence of type 2 diabetes and the association with abdominal obesity lead to growing health disparities in the USA: an analysis of the NHANES surveys from 1999 to 2014. Diabetes Obes Metab. 2018;20(3):667-671. doi:10.1111/dom. 13143

3. Coughlan KA, Valentine RJ, Ruderman NB, Saha AK. AMPK activation: a therapeutic target for type 2 diabetes?. Diabetes Metab Syndr Obes. 2014;7:241-253.

4. Foretz M, Hébrard S, Leclerc J, et al. Metformin inhibits hepatic gluconeogenesis in mice independently of the LKB1/AMPK pathway via a decrease in hepatic energy state. J Clin Invest. 2010;120 (7):2355-2369.

5. Chandel NS, Avizonis D, Reczek CR, et al. Are metformin doses used in murine cancer models clinically relevant?. Cell Metab. 2016;23(4):569-570.

6. Pollak M. The effects of metformin on gut microbiota and the immune system as research frontiers. Diabetologia. 2017;60 (9):1662-1667.

7. Burcelin R, Serino M, Chabo C, Blasco-Baque V, Amar J. Gut microbiota and diabetes: from pathogenesis to therapeutic perspective. Acta Diabetol. 2011;48(4):257-273.

8. Ley RE, Hamady M, Lozupone C, et al. Evolution of mammals and their gut microbes. Science. 2008;320(5883):1647-1651.

9. Bäckhed F. Host responses to the human microbiome. Nutr Rev. 2012;70(Suppl 1):S14-S17.

10. Larsen N, Vogensen FK, van den Berg FW, et al. Gut microbiota in human adults with type 2 diabetes differs from non-diabetic adults. PLoS One. 2010;5(2):e9085.

11. Qin J, Li Y, Cai Z, et al. A metagenome-wide association study of gut microbiota in type 2 diabetes. Nature. 2012;490(7418):55-60. doi:10.1038/nature 11450

12. Karlsson FH, Tremaroli V, Nookaew I, et al. Gut metagenome in European women with normal, impaired and diabetic glucose control. Nature. 2013;498(7452):99-103. doi:10.1038/nature12198

13. Cani PD, Bibiloni R, Knauf C, et al. Changes in gut microbiota control metabolic endotoxemia-induced inflammation in high-fat diet-induced obesity and diabetes in mice. Diabetes. 2008;57 (6):1470-1481.
14. Cani PD, Osto M, Geurts L, Everard A. Involvement of gut microbiota in the development of low-grade inflammation and type 2 diabetes associated with obesity. Gut Microbes. 2012;3(4):279-288. doi:10.4161/gmic. 19625

15. De Vadder F, Kovatcheva-Datchary P, Goncalves D, et al. Microbiota-generated metabolites promote metabolic benefits via gut-brain neural circuits. Cell. 2014;156(1-2):84-96. doi:10.1016/j. cell.2013.12.016

16. Koh A, De Vadder F, Kovatcheva-Datchary P, Bäckhed F. From dietary fiber to host physiology: short-chain fatty acids as key bacterial metabolites. Cell. 2016;165(6):1332. doi:10.1016/j.cell.2016.05.041

17. Yang XQ, Li LL, Wang Y. The association between the metabolites of intestional flora and diabetes mellitus in mice. Chin J Microecol. 2011;23(2):134-136.

18. Ryan PM, Stanton C, Caplice NM. Bile acids at the cross-roads of gut microbiome-host cardiometabolic interactions. Diabetol Metab Syndr. 2017;9:102.

19. Fava S. Glucagon-like peptide 1 and the cardiovascular system. Curr Diabetes Rev. 2014;10(5):302-310.

20. Pavlidis P, Powell N, Vincent RP, Ehrlich D, Bjarnason I, Hayee B. Systematic review: bile acids and intestinal inflammation-luminal aggressors or regulators of mucosal defence?. Aliment Pharmacol Ther. 2015;42(7):802-817.

21. Ridlon JM, Harris SC, Bhowmik S, Kang DJ, Hylemon PB. Consequences of bile salt biotransformations by intestinal bacteria. Gut Microbes. 2016;7(1):22-39. doi:10.1080/19490976.2015.1127483

22. Winston JA, Theriot CM. Impact of microbial derived secondary bile acids on colonization resistance against clostridium difficile in the gastrointestinal tract. Anaerobe. 2016;41:44-50.

23. Weingarden AR, Chen $\mathrm{C}$, Zhang $\mathrm{N}$, et al. Ursodeoxycholic acid inhibits clostridium difficile spore germination and vegetative growth, and prevents the recurrence of ileal pouchitis associated with the infection. J Clin Gastroenterol. 2016;50(8):624-630. doi:10.1097/MCG.0000000000000427

24. Sonne DP, van Nierop FS, Kulik W, Soeters MR, Vilsbøll T, Knop FK. Postprandial plasma concentrations of individual bile acids and FGF-19 in patients with type 2 diabetes. J Clin Endocrinol Metab. 2016;101 (8):3002-3009. doi:10.1210/jc.2016-1607

25. Zhang F, Yuan W, Wei Y, et al. The alterations of bile acids in rats with high-fat diet/streptozotocin-induced type 2 diabetes and their negative effects on glucose metabolism. Life Sci. 2019;229:80-92.

26. Shin NR, Lee JC, Lee HY, et al. An increase in the Akkermansia spp. population induced by metformin treatment improves glucose homeostasis in diet-induced obese mice. Gut. 2014;63(5):727-735. doi:10.1136/gutjnl-2012-303839

27. Lee H, Ko G. Effect of metformin on metabolic improvement and gut microbiota. Appl Environ Microbiol. 2014;80(19):5935-5943. doi:10.1128/AEM.01357-14

28. Bornstein S, Moschetta M, Kawano Y, et al. Metformin affects cortical bone mass and marrow adiposity in diet-induced obesity in male mice. Endocrinology. 2017;158(10):3369-3385.

29. Lee H, Lee Y, Kim J, et al. Modulation of the gut microbiota by metformin improves metabolic profiles in aged obese mice. Gut Microbes. 2018;9(2):155-165.

30. Wang J-H, Bose S, Shin NR, Chin Y-W, Choi YH, Kim H. Pharmaceutical impact of and metformin combination on high-fatdiet-induced metabolic disorders: link to intestinal microbiota and metabolic endotoxemia. Front Endocrinol (Lausanne). 2018;9:620.

31. Zheng J, Li H, Zhang X, et al. Prebiotic mannan-oligosaccharides augment the hypoglycemic effects of metformin in correlation with modulating gut microbiota. $J$ Agric Food Chem. 2018;66 (23):5821-5831. doi:10.1021/acs.jafc.8b00829

32. Ji S, Wang L, Li L. Effect of metformin on short-term high-fat diet-induced weight gain and anxiety-like behavior and the gut microbiota. Front Endocrinol (Lausanne). 2019;10:704. doi:10.33 89/fendo.2019.00704 
33. Brandt A, Hernández-Arriaga A, Kehm R, et al. Metformin attenuates the onset of non-alcoholic fatty liver disease and affects intestinal microbiota and barrier in small intestine. Sci Rep. 2019;9 (1):6668.

34. Ryan PM, Patterson E, Carafa I, et al. Metformin and dipeptidyl peptidase-4 inhibitor differentially modulate the intestinal microbiota and plasma metabolome of metabolically dysfunctional mice. Can J Diabetes. 2020;44(2):146-155.e2. doi:10.1016/j.jcjd.2019.05.008

35. Chung E, Elmassry MM, Kottapalli P, et al. Metabolic benefits of annatto-extracted tocotrienol on glucose homeostasis, inflammation, and gut microbiome. Nutr Res. 2020;77:97-107. doi:10.1016/j. nutres.2020.04.001

36. Ahmadi S, Razazan A, Nagpal R, et al. Metformin reduces aging-related leaky gut and improves cognitive function by beneficially modulating gut microbiome/goblet cell/mucin axis. J Gerontol a Biol Sci Med Sci. 2020;75(7):e9-e21.

37. Chen C, You LJ, Huang Q, et al. Modulation of gut microbiota by mulberry fruit polysaccharide treatment of obese diabetic $\mathrm{db} / \mathrm{db}$ mice. Food Funct. 2018;9(7):3732-3742.

38. Zhang W, Xu JH, Yu T, Chen QK. Effects of berberine and metformin on intestinal inflammation and gut microbiome composition in $\mathrm{db} / \mathrm{db}$ mice. Biomed Pharmacother. 2019;118:109131.

39. Gao K, Yang R, Zhang J, et al. Effects of Qijian mixture on type 2 diabetes assessed by metabonomics, gut microbiota and network pharmacology. Pharmacol Res. 2018;130:93-109. doi:10.1016/j. phrs.2018.01.011

40. Chen LC, Fan ZY, Wang HY, Wen DC, Zhang SY. Effect of polysaccharides from adlay seed on anti-diabetic and gut microbiota Food Funct. 2019;10(7):4372-4380.

41. Zhang $\mathrm{X}$, Zhao $\mathrm{Y}, \mathrm{Xu} \mathrm{J}$, et al. Modulation of gut microbiota by berberine and metformin during the treatment of high-fat diet-induced obesity in rats. Sci Rep. 2015;5:14405.

42. Li Q, He R, Zhang F, Zhang J, Lian S, Liu H. Combination of oligofructose and metformin alters the gut microbiota and improves metabolic profiles, contributing to the potentiated therapeutic effects on diet-induced obese animals. Front Endocrinol (Lausanne). 2019;10:939.

43. Liu G, Bei J, Liang L, Yu G, Li L, Li Q. Stachyose improves inflammation through modulating gut microbiota of high-fat diet/ streptozotocin-induced type 2 diabetes in rats. Mol Nutr Food Res. 2018;62(6):e1700954.

44. Liu G, Liang L, Yu G, Li Q. Pumpkin polysaccharide modifies the gut microbiota during alleviation of type 2 diabetes in rats. Int $\mathrm{J}$ Biol Macromol. 2018;115:711-717.

45. Cui HX, Zhang LS, Luo Y, Yuan K, Huang ZY, Guo Y. A purified anthraquinone-glycoside preparation from rhubarb ameliorates type 2 diabetes mellitus by modulating the gut microbiota and reducing inflammation. Front Microbiol. 2019;10:1423.

46. Liu Y, Wang C, Li J, et al. Phellinus linteus polysaccharide extract improves insulin resistance by regulating gut microbiota composition. FASEB J. 2020;34(1):1065-1078.

47. Khat-Udomkiri N, Toejing P, Sirilun S, Chaiyasut C, Lailerd N. Antihyperglycemic effect of rice husk derived xylooligosaccharides in high-fat diet and low-dose streptozotocin-induced type 2 diabetic rat model. Food Sci Nutr. 2020;8(1):428-444.

48. Wang JH, Bose S, Lim SK, et al. Houttuynia cordata facilitates metformin on ameliorating insulin resistance associated with gu microbiota alteration in OLETF rats. Genes (Basel). 2017;8(10):239.

49. Zhang M, Feng R, Yang M, et al. Effects of metformin, acarbose, and sitagliptin monotherapy on gut microbiota in Zucker diabetic fatty rats. BMJ Open Diabetes Res Care. 2019;7(1):e000717.

50. Hiel S, Gianfrancesco MA, Rodriguez J, et al. Link between gut microbiota and health outcomes in inulin -treated obese patients: lessons from the food4gut multicenter randomized placebo-controlled trial. Clin Nutr. 2020;S02615614(20):30160-30166
51. Ejtahed HS, Tito RY, Siadat SD, et al. Metformin induces weight loss associated with gut microbiota alteration in non-diabetic obese women: a randomized double-blind clinical trial. Eur J Endocrinol. 2019;180(3):165-176.

52. Forslund K, Hildebrand F, Nielsen T, et al. Disentangling type 2 diabetes and metformin treatment signatures in the human gut microbiota. Nature. 2015;528(7581):262-266.

53. Wu H, Esteve E, Tremaroli V, et al. Metformin alters the gut microbiome of individuals with treatment-naive type 2 diabetes, contributing to the therapeutic effects of the drug. Nat Med. 2017;23 (7):850-858.

54. de la Cuesta-zuluaga J, Mueller NT, Corrales-Agudelo V, et al. Metformin is associated with higher relative abundance of mucin-degrading akkermansia muciniphila and several short-chain fatty acid-producing microbiota in the gut. Diabetes Care. 2017;40 (1):54-62.

55. Sun L, Xie C, Wang G, et al. Gut microbiota and intestinal FXR mediate the clinical benefits of metformin. Nat Med. 2018;24 (12):1919-1929.

56. Hung F, Nilholm C, Roth B, et al. Anthropometric and metabolic improvements in human type 2 diabetes after introduction of an okinawan-based nordic diet are not associated with changes in microbial diversity or SCFA concentrations. Int J Food Sci Nutr. 2018;69 (6):729-740.

57. Barengolts E, Green SJ, Eisenberg Y, et al. Gut microbiota varies by opioid use, circulating leptin and oxytocin in African American men with diabetes and high burden of chronic disease. PLoS One. 2018;13 (3):e0194171.

58. Zhang F, Wang M, Yang J, et al. Response of gut microbiota in type 2 diabetes to hypoglycemic agents. Endocrine. 2019;66(3):485-493.

59. Chávez-Carbajal A, Pizano-Zárate ML, Hernández-Quiroz F, et al. Characterization of the gut microbiota of individuals at different T2D stages reveals a complex relationship with the host. Microorganisms. 2020;8(1):94.

60. Deng GH, Cha YL, Zhang GX, et al. Comparison of human and animal fecal microbiota with Illumina sequencing of 16S rRNA tags. Ecol Sci. 2014;33(5):851-857.

61. Ahmad A, Yang W, Chen G, et al. Analysis of gut microbiota of obese individuals with type 2 diabetes and healthy individuals. PLoS One. 2019;14(12):e0226372.

62. Sedighi M, Razavi S, Navab-Moghadam F, et al. Comparison of gut microbiota in adult patients with type 2 diabetes and healthy individuals. Microb Pathog. 2017;111:362-369.

63. Peng W, Huang J, Yang J, et al. Integrated 16S rRNA sequencing, metagenomics, and metabolomics to characterize gut microbial composition, function, and fecal metabolic phenotype in non-obese type 2 diabetic goto-kakizaki rats. Front Microbiol. 2019;10:3141.

64. Bibbò S, Ianiro G, Giorgio V, et al. The role of diet on gut microbiota composition. Eur Rev Med Pharmacol Sci. 2016;20 (22):4742-4749

65. Elbere I, Kalnina I, Silamikelis I, et al. Association of metformin administration with gut microbiome dysbiosis in healthy volunteers. PLoS One. 2018;13(9):e0204317.

66. Bryrup T, Thomsen CW, Kern T, et al. Metformin-induced changes of the gut microbiota in healthy young men: results of a non-blinded, one-armed intervention study. Diabetologia. 2019;62(6):1024-1035.

67. Ma W, Chen J, Meng Y, Yang J, Cui Q, Zhou Y. Metformin alters gut microbiota of healthy mice: implication for its potential role in gut microbiota homeostasis. Front Microbiol. 2018;9:1336.

68. Zhou ZY, Ren LW, Zhan P, Yang HY, Chai DD, Yu ZW. Metformin exerts glucose-lowering action in high-fat fed mice via attenuating endotoxemia and enhancing insulin signaling. Acta Pharmacol Sin. 2016;37(8):1063-1075.

69. Carter D, Howlett HC, Wiernsperger NF, Bailey CJ. Differential effects of metformin on bile salt absorption from the jejunum and ileum. Diabetes Obes Metab. 2003;5(2):120-125. 
70. Napolitano A, Miller S, Nicholls AW, et al. Novel gut-based pharmacology of metformin in patients with type 2 diabetes mellitus. PLoS One. 2014;9(7):e100778.

71. Zhang X, Shen D, Fang Z, et al. Human gut microbiota changes reveal the progression of glucose intolerance. PLoS One. 2013;8(8): e71108.

72. Cani PD, de Vos WM. Next-generation beneficial microbes: the case of akkermansia muciniphila. Front Microbiol. 2017;8:1765.

73. Delzenne NM, Cani PD, Everard A, Neyrinck AM, Bindels LB. Gut microorganisms as promising targets for the management of type 2 diabetes. Diabetologia. 2015;58(10):2206-2217.
74. Horakova O, Kroupova P, Bardova K, et al. Metformin acutely lowers blood glucose levels by inhibition of intestinal glucose transport. Sci Rep. 2019;9(1):6156.

75. Morita Y, Nogami M, Sakaguchi K, et al. Enhanced release of glucose into the intraluminal space of the intestine associated with metformin treatment as revealed by [(18)F]fluorodeoxyglucose PET-MRI. Diabetes Care. 2020;43(8):1796-1802.

76. Bauer PV, Duca FA, Waise TMZ, et al. Metformin alters upper small intestinal microbiota that impact a glucose-SGLT1-sensing glucoregulatory pathway. Cell Metab. 2018;27(1):101-117.e5.

\section{Publish your work in this journal}

Diabetes, Metabolic Syndrome and Obesity: Targets and Therapy is an international, peer-reviewed open-access journal committed to the rapid publication of the latest laboratory and clinical findings in the fields of diabetes, metabolic syndrome and obesity research. Original research, review, case reports, hypothesis formation, expert opinion and commentaries are all considered for publication. The manuscript management system is completely online and includes a very quick and fair peer-review system, which is all easy to use. Visit http://www.dovepress.com/testimonials.php to read real quotes from published authors. 